\title{
Tandem Duplications of the Histidine Operon Observed Following Generalized Transduction in Salmonella typhimurium
}

\author{
R. Philip Anderson†, Charles G. Millert and John R. Roth $\dagger$ \\ Department of Molecular Biology, University of California \\ Berkeley, Calif. 94720. U.S.A.
}

(Received 12 January 1976)

\begin{abstract}
Unstable merodiploid transductants may be observed among the progeny of certain generalized transductional crosses between complementing mutations in the histidine operon of Salmonella typhimurium. In the presence of a functional recombination system, these transductants are unstable and they segregate $\mathrm{His}^{-}$ clones of both parental genotypes. The properties of these His ${ }^{+}$transductants suggest that they contain tandem duplications of a region of DNA which includes the histidine operon, such that each copy of the duplication contains one of the two complementing mutations involved in the transduction. Transductional duplications have been observed from 14 pairs of his mutations, but only with complementing pairs of parental mutations. The length of duplicated material may be quile large: two duplications were found to include genetic markers ten minutes removed from the histidine operon on the Salmonella chromosomal map.

These transductants appoar to arise in a subpopulation of recipient cells which contain pre-existing tandem duplications of the histidine operon. As much as 0.01 to $0.1 \%$ of the cell population appears to be tandemly duplicated for a ehromosomal region which includes the histidine operon.
\end{abstract}

\section{Introduction}

Tandem genetic duplications have been observed in bacteria under a variety of circumstances. Recently published data have suggested that spontaneous formation of tandem duplications is, in fact, a quite common event. Duplication rates of approximately $10^{-5}$ have been reported for the glyS gene (glycyl-transfer RNA synthetase, Folk \& Berg, 1971), the $\arg H$ gene $(N$-acetyl- $\gamma$-semialdehyde dehydrogenase, Glansdorff \& Sand, 1968), and the gene(s) required for L-malate utilization (Straus \& Hoffmann, 1975). Studies involving a known informational missense suppressor $\left(g l y T s u_{A G A}\right)$ indicate that $10 \%$ of induced suppressors are tandemly diploid for the gly $T$ locus (Hill \& Combriato, 1973), and presumably arise in strains carrying preexisting duplications of the $g l y T$ region. Moreover, after extremely mild u.v. irradiation ( $75 \%$ survival), fully 4 to $5 \%$ of surviving chromosomes harbor tandem duplications of the glyT-purD region. Tranductional studies of the Salmonella lethal suppressor mutations, $\sup R$ and $\sup S$, suggest that spontaneous duplications of that chromosomal region may be carried by greater than $10 \%$ of the cell population (Miller \& Roth, 1971).

† Jresent address: Department of Biology, University of Utah, Salt Lake City, Utah 84112, U.S.A.

$\ddagger$ Present address: Department of Microbiology, ('ase Western Reserve School of Medicine, Cleveland, Ohio 44106, U.S.A. 
Although observed by a variety of genetic techniques and maintained by a variety of selective pressures, all of these genetic duplications share a common property-their instability. In the absence of selection for the partially diploid state, haploid segregants are observed at high frequency-most likely duc to recombination between tandemly located homologous regions of the same chromosome.

To study the process of gene duplication and to provide an assay for duplication frequencies, we have designed a method for detection of duplications in a genctically well-studied region of the Salmonella chromosome, the histidine operon (Hartman et al., 1971). Tandem duplications of the histidine operon may be observed as a class of unstable transductant progeny arising from standard transductional crosses involving complementing his mutations. They are most easily observed when the production of normal wild-type (haploid) transductants is severely restricted. This is accomplished by crossing complementing, non-overlapping histidine deletions which contain a very limited region of homology between their endpoints. The duplication strains are quite unstable, and accumulate His ${ }^{-}$haploid segregants of both parental genotypes. This paper describes the formation and characteristics of these transductional duplications.

\section{Materials and Methods}

(a) Media and growth conditions

Vogel \& Bonner (1956) E medium containing $2 \%$ glucose was used as minimal medium. When required, this medium was supplemented with $0.1 \mathrm{~mm}$-(excess) or 0.005 mu-(limiting) histidine, 1.0 mm-hislidinol, $4 \mathrm{mM}$-serine, $0.5 \mathrm{~mm}$-adenine, and approximately $0.3 \mathrm{~mm}$ other amino acids. DL-amino acids were used, but the concentrations given are for the L-isomer. When used, 3-amino-1,2,4-triazole (Aldrich) was included at $20 \mathrm{~mm}$. Difco nutriont broth $(0.8 \%)$ containing $0.5 \% \mathrm{NaCl}$ was used as complex medium. Solid media contained $2 \%$ Difco agar. All incubations were performed at $37^{\circ} \mathrm{C}$ with aeration by gyratory shaking.

\section{(b) Bacterial strains}

All strains are derivatives of S. typhimurium strain LT-2. Relevant genotypes are presented in the text. All his deletion mutants were obtained from P. E. Hartman or B. N. Ames.

\section{(i) Transduction}

(c) Genetic techniques

The non-lysogenizing P22 mutant int-4 (Smith \& Levine, 1967) was used in all transductions. Plates were spread with a mixture of $2 \times 10^{8}$ to $6 \times 10^{8}$ recipient cells and approximately $10^{9}$ phage particles. Transductant clones were scored after 2 to 4 days' incubation at $37^{\circ} \mathrm{C}$. When transductants were to be used in further work, clones were purified 3 times selectively, and once non-selectively. They were verified to be nonlysogenic (see below) and preserved.

\section{(ii) Preservation of strains}

Cultures of unstable duplication strains in minimal medium were supplemented to contain $8 \%$ dimethyl sulfoxide (Matheson, Coleman, and Bell Co.) and frozen at $-70^{\circ} \mathrm{C}$ in a Kelvinator Ultra Cold Freezer. We have found this technique exceptionally good for long-term preservation of strains whose desired genetic determinants are unstable (duplications, $F^{\prime}$ episomes, Hfr strains, P22 lysogens, etc.).

\section{(iii) Hfr transfer}

Stationary phase cultures of $\mathbf{H f r}$ donors were diluted 100 -fold into frosh nutriont media and grown with aeration for $2 \mathrm{~h}: 0 \cdot 1 \mathrm{-ml}$ samples (approx. $10^{7}$ cells) were then mixed with $2 \times 10^{8}$ to $6 \times 10^{8}$ recipient cells and plated on appropriate solid media. Conjugants 
were scored after 2 days incubation at $37^{\circ} \mathrm{C}$. Representative clones were picked, purified selectively once, and retested for the desired genotype. Recombination deficiency (recA ${ }^{-}$) was scored as sensitivity to $400 \mathrm{erg} / \mathrm{mm}^{2}$ of ultraviolet light irradiation.

\section{(iv) Phage sensitivity}

To test for the presence of cryptic phage genes in transductants, drops of lysates $\left(\sim 10^{6}\right.$ plaque-forming units/ml) of P22 wild-type (Levine, 1972), P22 H5 (a clear-plaque mutant; Zinder, 1958), and numerous P22 amber mutants (Lew \& Roth, 1970; Botstein et al., 1972) defective in genes throughout the phage genome were spotted onto soft agar lawns of the strains to be tested. Under these conditions, lysis by both wild type and H5 phage indicates P22 sensitivity, and lysis by mutant phage indicates recombination between cryptic and superinfecting phage genomes.

\section{(v) Segregation analysis}

For determination of segregant frequencies, a single colony of an extensively purified duplication strain (see text) was picked from selective medium and grown 10 to 15 generations in liquid nutrient broth. Cultures were then diluted, and spread for single colonies on plates containing a limiting amount of histidine $(0.005 \mathrm{~mm})$. Under these conditions, $\mathrm{His}^{+}$clones form large, round colonies, and $\mathrm{His}^{-}$segregant clones form small, flat, easily distinguishable colonies. Parental histidine mutations in the His - segregants were identified by the ability $\left(\right.$ his $\left.D^{+}\right)$or inability $\left(\right.$his $\left.D^{-}\right)$to utilize L-histidinol as a source of histidine following 3 days incubation at $37^{\circ} \mathrm{C}$. For segregation analysis, parental deletion mutations were chosen such that one was his $D^{+}$and one hisD.

\section{Results}

\section{(a) Observation of merodiploid transductants}

Among the progeny of certain generalized transductional crosses in the histidine operon of Salmonella are a class of highly unstable merodiploid transductants. Figure 1 shows the number of His + transductants formed in reciprocal crosses between the complementing, non-overlapping histidine deletions $h i s-2236$ and his-2527. The severe reduction in total prototrophic transductants (as compared to a wild-type LT.2 donor) is, of course, due to the necessity of a recombinational event in the small region which separates these two mutations. When other more closely linked histidine
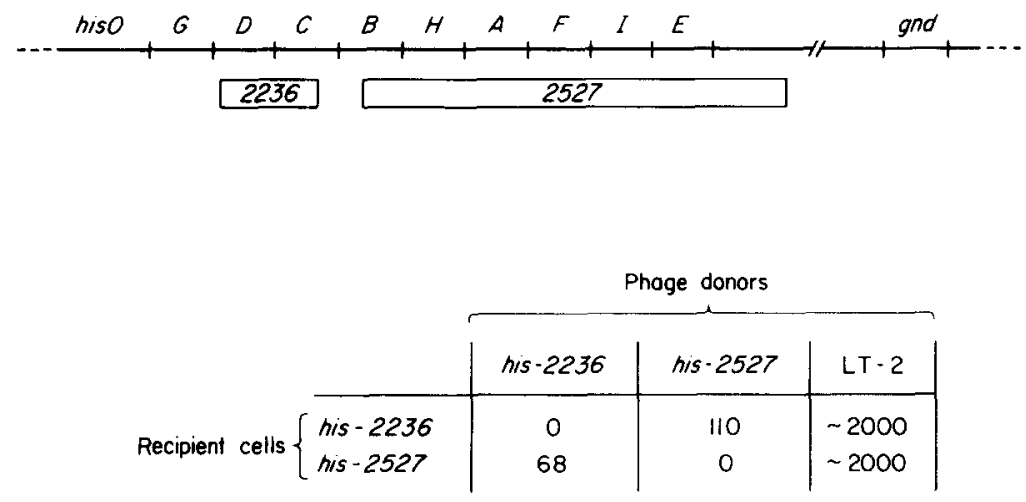

FIG. 1. Prototrophic recombinants obtained following reciprocal transductional crosses between complementing, non-overlapping histidine deletions his-2236 and his.2527. Crosses were performed as described in Materials and Methods. 
deletion pairs are crossed, the reduction can be even more severe. Among the prototrophic recombinants which arise in such crosses are merodiploid transductants which retain the recipient deletion mutation and inherit (by addition) a second histidine region harboring the complementing donor mutation. Depending upon the pair of complementing histidine deletions crossed, merodiploids can constitute a varying fraction of total prototrophs, ranging from a few to $100 \%$.

Many of these merodiploid transductants form mucoid colonies on minimal medium. This is most likely due to the presence of some gene (or genes) near the histidine operon, which, when present in diploid or greater amount, renders the cell mucoid. In Escherichia coli this mucoid characteristic has been observed in strains which carry certain $\mathrm{F}^{\prime}$ his episomes. and thus possess multiple copies of the chromosomal region surrounding the histidine operon (Silverman \& Simon, 1973). This mucoid character is quite helpful in identifying merodiploids among wild-type recombinants. It is an easily identifiable trait exhibited by many (but not all) $\mathrm{His}^{+}$merodiploid transductants.

\section{(b) Instability of merodiploids}

These merodiploid transductants are unstable. When grown non-selectively (in the presence of excess histidine), cultures of these $\mathrm{His}^{+}$strains accumulate $\mathrm{His}^{-}$segregants of both parental deletion types at high frequency. Following 10 to 15 generations of growth in non-selective media, a culture may contain 5 to $75 \%$ His ${ }^{-}$segregants. The two parental his ${ }^{-}$mutations which constitute this segregant population

\section{TABLE 1}

Haploid parental segregants from unstable merodiploid transductants

\begin{tabular}{|c|c|c|}
\hline Merodiploid formation & \multicolumn{2}{|c|}{ Haploid segregant types (percent) } \\
\hline Phage donor $\times$ Recipient cells: & $h i s-2236$ & his-2527 \\
\hline his-2236 $\times$ his-2527 & $24 \%(11 / 46)$ & $76 \%(35 / 46)$ \\
\hline his-2236 $\times$ his-2527 & $26 \quad(13 / 49)$ & $(36 / 49)$ \\
\hline his-2236 $\times$ his-2527 & $(14 / 49)$ & $(35 / 49)$ \\
\hline his-2236 $\times$ his.2527 & $(17 / 45)$ & $(28 / 45)$ \\
\hline his-2236 $\times$ his. 2527 & $(26 / 47)$ & $(21 / 47)$ \\
\hline his-2236 $\times$ his-2527 & $(33 / 46)$ & $(13 / 46)$ \\
\hline his- $2236 \times h i s-2527$ & $(33 / 48)$ & $(13 / 48)$ \\
\hline his. $2236 \times h i s-2527$ & $(38 / 49)$ & $(11 / 49)$ \\
\hline his-2236 $\times$ his. 2527 & $90 \quad(41 / 46)$ & $(5 / 46)$ \\
\hline his-2527 $\times$ his-2236 & $16 \% \quad(8 / 48)$ & $84 \%(40 / 48)$ \\
\hline$h i s \cdot 2527 \times h i s-2236^{\circ}$ & $24 \quad(11 / 46)$ & $76 \quad(35 / 46)$ \\
\hline his-2527 $\times$ hi.s-2236 & $(12 / 46)$ & $(34 / 46)$ \\
\hline$h i s-2527 \times h i s-2236$ & $(14 / 48)$ & $(34 / 48)$ \\
\hline his-2527 $\times$ his-2236 & $(18 / 43)$ & $(25 / 43)$ \\
\hline his-2527 $\times$ his- 2236 & $(32 / 50)$ & $(18 / 50)$ \\
\hline his-2527 $\times$ his-2236 & $(30 / 45)$ & $(15 / 45)$ \\
\hline his.2527 $\times$ his-2236 & $(38 / 50)$ & $(12 / 50)$ \\
\hline his-2527 $\times$ his-2236 & $(41 / 49)$ & $(8 / 49)$ \\
\hline his. $2527 \times$ his -2236 & $(41 / 49)$ & $(8 / 49)$ \\
\hline
\end{tabular}

All merodiploids are independent isolates from transductional crosses performed as indicated. Extensively purified transductant clones were grown non-selectively and $\mathrm{His}_{\text {- haploid parental }}$ segregants were identified as described in Materials and Methods. Figures in parentheses are the actual data. For each merodiploid, similar segregation results have been obtained in repeat experiments. 
are present in various ratios, but each merodiploid transductant exhibits a characteristic and reproducible ratio of parental genotypes among its segregant progeny. Segregation data from 19 independent merodiploid isolates generated by crosses involving deletions his-2236 and his-2527 are shown in Table 1 . For each isolate, the frequencies of segregant types are expressed as percentage of total His ${ }^{-}$segregants following 10 to 15 generations of non-selective growth. A variety of segregant frequencies is evident. There is no tendency to recover merodiploid clones exhibiting any particular segregation behavior.

The extensive purification of merodiploid transductants tested in the above manner insures that they are single bacterial clones and not mixed populations of crossfeeding haploid auxotrophs. Transductant clones have been isolated as single colonies three times selectively, once non-selectively (nutrient agar), and once again selectively before segregation analysis. The fact that phenotypically His ${ }^{+}$clones can be isolated non selectively argues strongly that the $\mathrm{His}^{+}$phenotype is expressed by single bacterial cells. Genetic experiments described later demonstrate clearly the merodiploid nature of these clones.

\section{(c) Proposed structure of these merodiploid transductants}

We feel that these unstable merodiploid transductants can best be explained as carrying tandem duplications of a region of chromosomal material which includes the histidine operon, such that each of the two histidine operons harbors one of the two complementing deletion mutations involved in the initial transduction. This model is described in more detail below. Experiments designed to test the model and to provide information about the mechanism of formation of these transductional duplications are presented in following sections.

Our model is depicted in Figure 2. The "squiggly" lines represent endpoints of a tandemly duplicated region which includes the histidine operon. The "dot" represents the junction between duplicated regions. Each copy of the duplication contains one
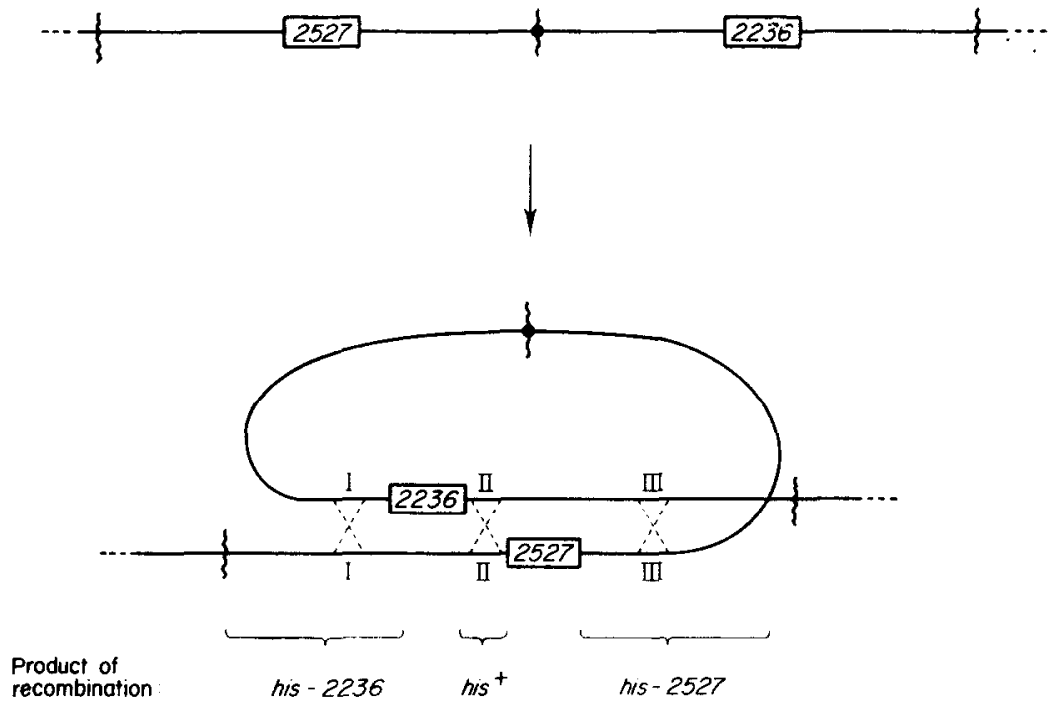

FIG. 2. A proposed molecular description for unstable merodiploid transductants. Histidine deletion mutations are represented by their allele numbers. Synapsis of tandemly located homologous sequences yields 3 paired regions, designated I, II and III. 
of the two complementing his deletions. (The Figure is not drawn to scale; duplications may be large relative to the size of deletions.) Because of tandemly located homologous sequences, such a structure can circularize, and by single recombinational events produce three haploid segregant types: both parental types and wild-type. A fourth segregant type, the double mutant, may be formed by a triple crossover event, in regions I, II and III. It should be noted that if a duplication strain carries the two mutant histidine operons in an opposite orientation to that depicted in Figure 2 the rare segregant type (generated by a single recombination between the deletion endpoints) is the double mutant, rather than the wild-type. The relative frequency with which each segregant type arises should be determined by the structure of the duplication. For the duplication shown in Figure 2, the relative frequencies of hap. loid segregant types should be determined by the relative lengths of regions I, II and III. The segregation data (see Table 1), therefore, suggest that independent isolates of transductional duplications arise with various structures-that is with various endpoints and sizes of duplicated regions. Wild-type segregants have been observed from some of our duplication strains, and will be discussed below. As might be expected, they occur at quite low frequencies (since they require a recombinational event in the small region of homology between the deletion endpoints).

\section{(d) Instability is dependent upon recombination}

Tandem genetic duplications are expected to generate haploid parental segregants as a result of circularization and recombination between internally homologous chromosomal regions (Campbell, 1963; see Fig. 2). Thus, duplications should be unstable only in recombination proficient bacteria. An experiment designed to test our putative duplications for this prediction is diagrammed in Figure 3. A Rec ${ }^{+}$ duplication strain has been constructed which is $\operatorname{ser} A^{-}$(the $\operatorname{ser} A$ locus is linked to rec $A$ in conjugational crosses). In the first cross shown, a rec $A^{-} l y s^{-}$Hfr donor (TR3261) was mated with this unstable duplication strain, selecting Ser $^{+}$. The Ser ${ }^{+}$
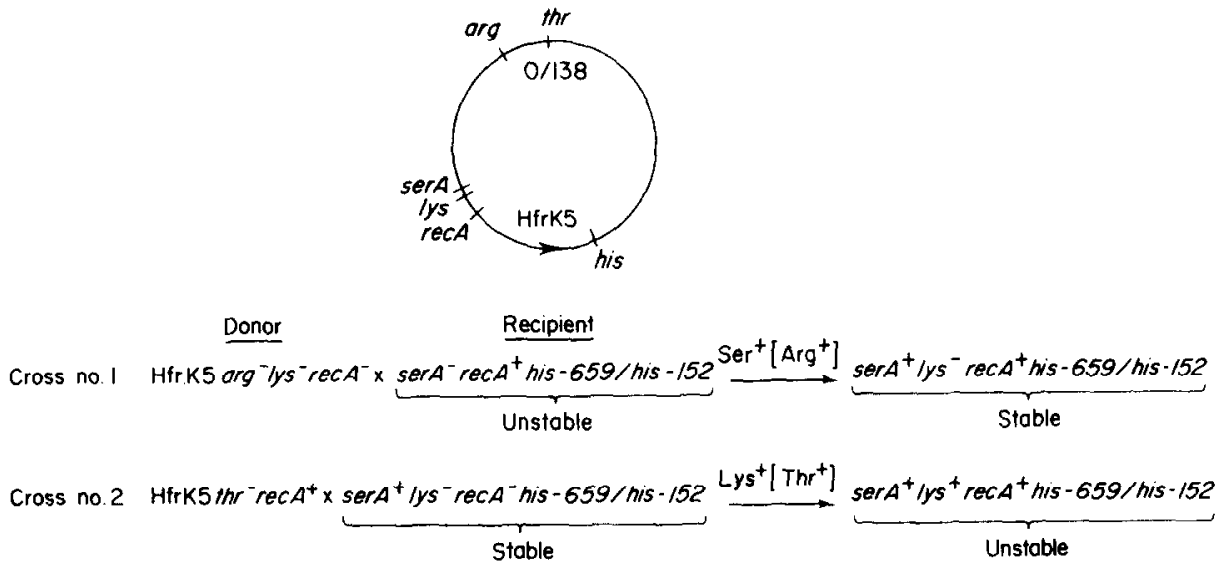

FIa. 3. Conjugational crosses indicating that instability of transductional duplications is dependent upon recombination function. Relevant genetic loci are indicated on the linkage map of S. typhimurium (Sanderson, 1972). All crosses were performed as described in Materials and Methods. Selected and counter-selected markers are indicated above the arrows as un-bracketed and bracketed phenotypes, respectively. Donor strains used were TR3261 (HfrK5 lys528 ary 1820 rec $A 1$ ) and TK217 (HfrK5 thr609 aroD5 his'T 1529). 
recombinants which retained the duplication were identified by their mucoid character, and the rec $A^{-} l y s^{-}$recombinants were obtained. All such strains were found to be stably $\mathrm{His}^{+}$. Following non-selective growth in rich medium, no $\mathrm{His}^{-}$haploid segregants were observed $\left(5 \times 10^{4}\right.$ clones tested). Under the same conditions, the $\mathrm{Rec}^{+}$parental duplication strain (the recipient in cross number 1) generated nearly $50 \% \mathrm{His}^{-}$segregants.

The second cross shown in Figure 3 demonstrates that these stable strains do. in fact. still harbor the duplication. In this cross, a rec $A^{+} \mathrm{Hfr}$ donor (TK217) was mated with one of the $\mathrm{Rec}^{-}$stable duplications, selecting Lys ${ }^{+}$recombinants. The Lys ${ }^{+}$ $\mathrm{Rec}^{+}$duplication recombinants were obtained, and were found to be unstably His ${ }^{+}$. Moreover, they segregated parental his deletion types in the same ratio characteristic of the original duplication strain. Such stabilization by recombination deficiency is expected of strains carrying tandem duplications of chromosomal material.

\section{(e) Frequency of transductional duplications}

In most crosses, these duplication transductants are quite rare. In crosses involving tightly linked mutations, however, the close proximity of these mutations limits the frequency of normal wild-type recombinants. Under these restrictive conditions, the relatively rare unstable class can represent an appreciable fraction of transductants which arise. Extrapolation from the frequency of duplications among these transductants and the frequency of transductants using a wild-type $\left(h \mathrm{is}^{+}\right)$donor permits us to estimate that following any cross within the histidine region (irrespective of restrictions upon recombination caused by tightly linked mutations), one transductant in $10^{3}$ to $10^{4}$ may involve duplication events such as those described here.

\section{(f) Generality of tranductional duplications}

To demonstrate that transduction to duplication is not an isolated phenomenon, we have crossed many different pairs of histidine deletions, and have found merodiploid transductants from 14 such pairs. Pairs of histidine deletions were chosen for testing which should complement each other (based upon the genetic map) and which have a limited region of homology between their endpoints. The use of tightly linked mutations reduced the number of wild-type prototrophic recombinants, such that the minority duplication types could be observed as a reasonable fraction of total prototrophs. The map positions of various histidine deletion pairs which formed transduc. tional duplications are shown in Figure 4. Duplications are observed when crossing many deletion pairs; and these deletion pairs are found widely scattered on the histidine operon genetic map.

Although these indicated 14 deletion pairs generated merodiploid transductants, 16 additional pairs, whose endpoints were also in different genes, did not yield unstable transductants. Our failure to find duplication transductants from these pairs is possibly due to two factors. Firstly, polarity effects (Franklin \& Luria, 1961 ; Jacob \& Monod, 1961) exhibited by these deletions may limit complementation. Many of the deletions used in this study were isolated by a procedure selecting directly for polar histidine mutations (Fink et al., 1967). Since histidine prototrophy is selected during transduction, the formation and identification of a duplication strain requires that the deletions in question complement sufficiently to allow colony formation. Weak complementation (due to strong polarity effects) may prevent detection of some duplications. 


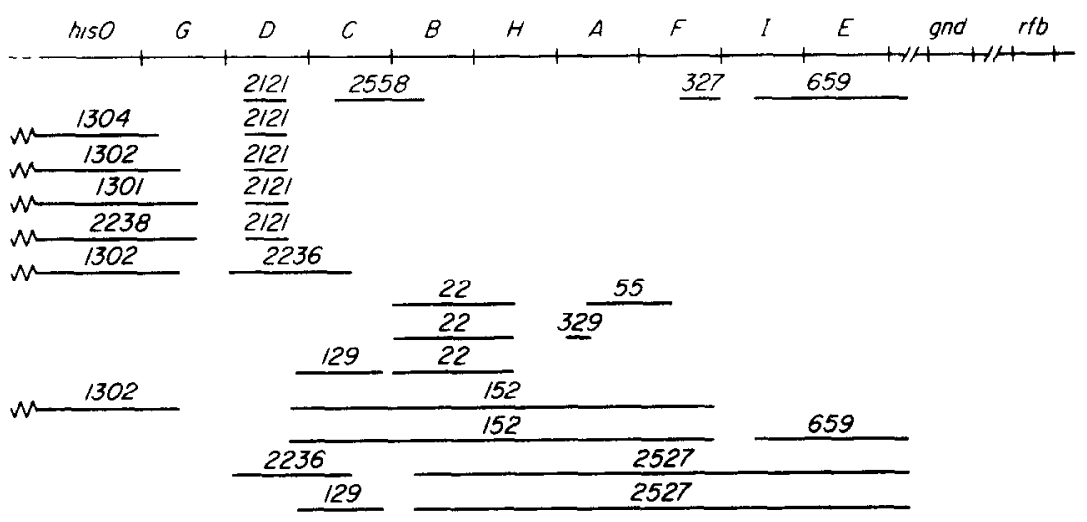

Fig. 4. Histidine deletion pairs which were found to generate unstable duplication transductants. All pairs affect different his genes. Histidine deletions 1301,1302, 1304 and 2238 maintain expression of unaffected genes, even though they are deleted for the promoter-operator region.

Secondly, the initial identification of duplication transductants relies to a moderate extent upon their being mucoid. The mucoid character allows merodiploid transductants to be easily identified among wild-type prototrophic recombinants. Thus, if duplications do not cause mucoidy, they may be overlooked. Small numbers of nonmucoid transductants were routinely tested for stability, and occasionally duplications were identified in this manner. Thus, it is possible that many duplications were overlooked due to their non-mucoid character. Such lack of mucoidy in duplication transductants may be due to several factors. Some strains may favor the formation of duplications which do not include the mucoidy determinants, or there may be some strain dependence of the mucoid character.

A significant characteristic of this phenomenon is that complementation between parental mutations is required for the observation of merodiploid transductants. Twenty additional deletion pairs which can recombine but which do not complement (the parental mutations affect a common gene) have been tested for the production of unstable transductants. None have been found.

Deletions themselves are not required for the formation of these tranductional duplications. We have observed unstable transductants from crosses involving complementing histidine point mutations or involving a point mutation and a deletion. In fact, it is even unnecessary to limit the production of wild-type recombinants by crossing tightly linked mutations. We have also observed duplication transductants using wild-type (LT-2) phage donor crossed with deletions. Such transductants exhibit the same instability characteristic of previously discussed tandem duplications. However, because of their segregation pattern, one copy is concluded to be wild-type and the other copy to harbor the recipient deletion mutation. As before, His ${ }^{-}$haploid segregants are formed at high frequency. However, in addition to $\mathrm{His}^{-}$haploid segregants, $\mathrm{His}^{+}$wild-type haploid segregants are also formed at high frequency. The $\mathrm{His}^{+}$segregants are considered to be wild-type because they are not mucoid and are stably $\mathrm{His}^{+}$, i.e. they do not give rise to $\mathrm{His}^{-}$segregant progeny. Such duplications have been identified by their mucoid character. When deletions his-2236 or his-2527 are transduced to His ${ }^{+}$with phage grown on wild-type Salmonella, approximately 0.01 to $0.1 \%$ of total His ${ }^{+}$transductants are mucoid. The mucoid transductants 
may be picked and tested for stability of their His ${ }^{+}$character. Somewhat less than half of the mucoid transductants obtained in this manner are unstable. (Some His ${ }^{+}$ transductants are mucoid for other, unknown reasons.)

\section{(g) Wild-type segregants}

As mentioned above, wild-type segregants have been observed from some of the duplication strains. Wild-type segregants from a typical duplication strain would be expected to be $\mathrm{His}^{+}$, non-mucoid and stable. Moreover, they should be quite rare, since the generation of a wild-type segregant requires a recombinational event in the small region between deletion endpoints (see Fig. 2). Several independently isolated duplications have been grown non-selectively and plated for single colonies on minimal medium No clones have been observed which meet the above criteria for being wild type. The following selection, however, has permitted the observation of wildtype segregants.

The histidine analog 3-amino-1,2,4-triazole is a specific inhibitor of the imidazole glycerol phosphate dehydratase activity of the hisB enzyme (Hilton et al., 1965). However, wild-type strains can overcome the growth inhibitory effects of this drug by derepression of the histidine operon. Duplications have been generated by crossing appropriately chosen deletion pairs such that: (1) one of the deletions includes the his $B$ gene; and (2) the other deletion is operator proximal to and strongly polar upon the $h i s B$ gene. The $h i s-2236 / h i s-2527$ duplication is an example of such a strain. Deletion his-2236 was isolated by the technique of direct selection for polar histidine mutations (Fink et al., 1967); deletion his-2527 deletes the IGP-dehydratase portion of the hisB gene. The his-2236/his-2527 duplication strain is sensitive to aminotriazole inhibition because it carries only one copy of the $h i s B$ gene, which due to polarity is unable to fully derepress. In order to escape aminotriazole inhibition, this strain must increase its level of his $B$ enzyme. Wild-type segregants should be aminotriazoleresistant, because they will have reconstructed an intact histidine operon and will be able to derepress sufficiently. Thus, the selection for wild-type segregants (following non-selective growth) is for histidine prototrophy and aminotriazole resistance. This selection has been performed on two duplication strains. In each case, both mucoid and non-mucoid $\mathrm{His}^{+} \mathrm{AT}^{\mathrm{R}}$ colonies arose. Upon further testing, the mucoid colonies were found to be unstable and the non-mucoid colonies stable. The mucoid isolates are possibly strains whose aminotriazole resistance is due to an increase in gene dosage. Once a tandem duplication has been generated, it is possible to increase the number of copies of the duplicated segment by means of "legitimate" recombination. Sister chromatids may synapse in a staggered fashion and recombine to produce a triplication and a haploid (Sturtevant, 1925; Parma et al., 1972). Further characterization of these strains has not yet been performed. The non-mucoid isolates have been shown to be wild-type because: (1) they are $\mathrm{His}^{+}$; (2) they are aminotriazole-resistant, (3) they are not mucoid, (4) they are stable and form no His - segregants following nonselective growth $(<0.01 \%)$, and (5) they are able to donate an intact histidine region. to recipients carrying a large his deletion, and do so with a frequency similar to that of wild-type donors. The $\mathrm{His}^{+}$duplication strains from which these wild-type segregants arose are unable to donate their $\mathrm{His}^{+}$character (or do so at extremely low frequency). Using the procedure outlined above, wild-type progeny have been estimated to constitute 0.01 to $0.001 \%$ of total haploid segregants following non-selective growth for both duplication strains tested. 


\section{(h) Non-involvement of specialized transduction}

Stodolsky (1973) has described a procedure similar to the one used here for the selection of $\mathrm{Pl}$-specialized transducing phage in $E$, coli. His procedure involves transductional crosses between complementing, tightly linked mutations using Pl-lysogenic recipients. Induction of the recipient's prophage during transduction is thought to result in breakage and reconstruction of DNA fragments, and specialized phage are generated which have incorporated the selected donor marker. The specialized phage then establish a complementing merodiploid merely by lysogenizing the recipient cells. In our experiments, it was possible that a low level of P22.his specialized phage was present in the initial donor lysate, and that we had selected for lysogeny by such phage. Four lines of evidence argue against this explanation. (I) All of our duplication transductants are non-lysogenic and $\mathrm{P} 22$-sensitive; thus, no phage immunity systems are evident. (2) None of the duplications plate P22 amber mutants; thus, no cryptic phage genes can be detected in the transductants. P22 mutants tested have included those from genes $1,2,4,5,8,19$ and 20. (3) We have used a non-integrating mutant of phage P22 (P22int-4) in our transductional crosses; thus, the site-specific integration system does not seem to be essential. In addition, duplications are generated at near-normal frequencies in strains carrying a deletion of the P22 prophage attachment site (data not shown). (4) Perhaps the strongest argument against specialized transduction is the large size of duplicated regions. Some transductants carry duplications which are much larger than material that could be packaged by phage P22. Evidence for this is presented below (see section (j)).

\section{(i) Transducibility of duplications}

Hill et al. (1969) have demonstrated how tandem genetic duplications may be transduced by phage $\mathrm{Pl}$, even though the duplicated material is larger than that which phage Pl can package. The elements of their model are presented in Figure 5. A critical requirement is that the selected donor marker(s) within the duplication (any of the capital letters in Fig. 5) and the connecting point between duplicated segments be cotransducible. Once this requirement is met, recombination events involving the transduced fragment and two sister chromatids in the recipient cell can regenerate the duplication state of the donor. In such transductants, much of the duplicated material is of recipient origin. Although alternative models may also account for all available data, their essential elements are the same: the diploid state of large genetic duplications may be transduced provided that the selected donor marker and the duplication junction are cotransducible.

It was of interest to determine whether any of our transductional duplications were themselves transducible. Seventeen independent isolates of duplications generated from crosses involving his-2236 and his-2527 were used as transductional donors in crosses described in Table 2. If a duplication is structured such that one of the parental deletions is co-transducible with the duplication junction, it should be an efficient donor for re-establishment of the duplication when a complementing deletion is used as the recipient. In such a case, regeneration of the duplication will occur by addition of the donor mutation to the recipient chromosome, thus re-establishing the complementing diploid state. If a duplication is small enough that both parental deletions are cotransducible (including the connecting point between them), it should be an efficient donor for re-establishment of the duplication state even when the 

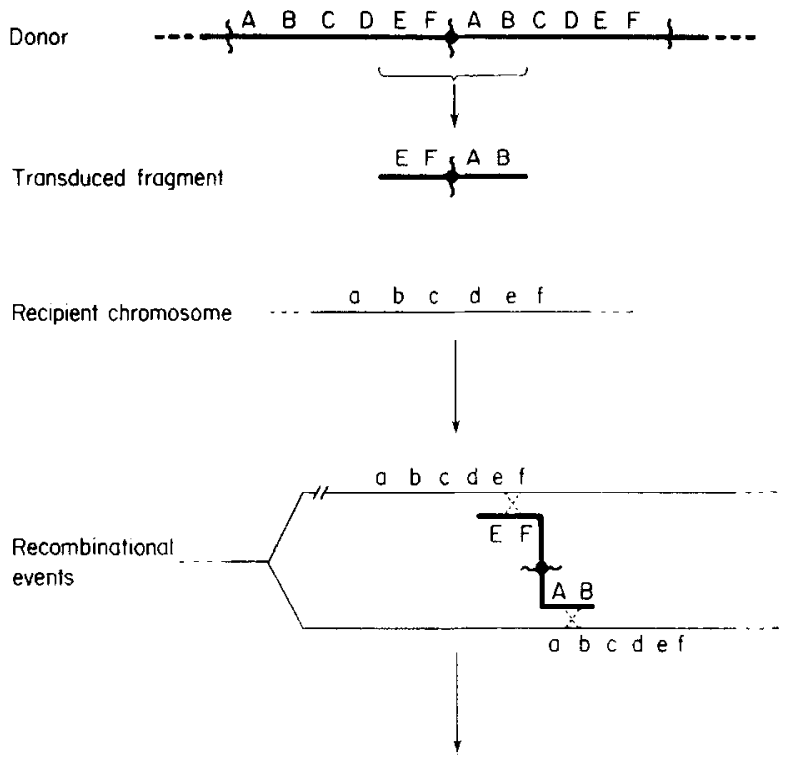

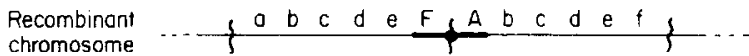

Fic. 5. A proposed mechanism for transducibility of tandem genetic duplications (Hill et al., 1969). Upper and lower case letters are non-genetic indications of hypothetical base sequences for donor (bold lined) and recipient (light lincd) DNAs, respectively. Solid lines indicate doublestranded DNA. Dashed lines represent reciprocal recombinational events.

TABLE 2

Non-transducibility of the duplication state

\begin{tabular}{|c|c|c|c|}
\hline \multirow{2}{*}{$\begin{array}{l}\text { Phage } \\
\text { donor }\end{array}$} & \multicolumn{3}{|c|}{$\begin{array}{c}\text { [Transductants/plaque-forming unit }] \times 10^{8} \\
\text { Recipient oolls }\end{array}$} \\
\hline & $h i s-2527$ & his-2236 & $h i s-152$ \\
\hline TR3612 & $2 \cdot 3$ & $3 \cdot 9$ & $<0.08$ \\
\hline TR3613 & $1 \cdot 1$ & $2 \cdot 2$ & $<0.08$ \\
\hline TR3614 & $1 \cdot 2$ & $3 \cdot 3$ & $<0.08$ \\
\hline TR3615 & 0.5 & $2 \cdot 3$ & $<0.08$ \\
\hline TR3616 & 0.6 & $2 \cdot 2$ & $<0.08$ \\
\hline 'IR3617 & $1 \cdot 8$ & $1 \cdot 1$ & $<0.08$ \\
\hline TR3618 & $2 \cdot 5$ & $1 \cdot 6$ & $<0.08$ \\
\hline TR3619 & $2 \cdot 5$ & $0 \cdot 8$ & $<0.08$ \\
\hline TR3620 & 0.5 & $2 \cdot 3$ & $<0.08$ \\
\hline TR3621 & $3 \cdot 2$ & $1 \cdot 2$ & $<0.08$ \\
\hline TR3622 & $3 \cdot 0$ & $2 \cdot 3$ & $<0.08$ \\
\hline TR3623 & $4 \cdot 7$ & $2 \cdot 5$ & $<0.08$ \\
\hline TR3624 & 3.8 & $2 \cdot 2$ & $<0.08$ \\
\hline TR3625 & $3 \cdot 1$ & 0.7 & $<0.08$ \\
\hline TR3626 & $0 \cdot 2$ & $1 \cdot 0$ & $<0.08$ \\
\hline TR3627 & 0.6 & $4 \cdot 4$ & $<0.08$ \\
\hline TR3628 & $3 \cdot 9$ & 2.11 & $<0.08$ \\
\hline his-2236 & $3 \cdot 0$ & $=0.118$ & $<0.08$ \\
\hline his-2527 & $<0.08$ & 1.5 & $n t$ \\
\hline LT-2 & 75 & 75 & 75 \\
\hline
\end{tabular}

Strains TR3612 through TR3628 are $\mathrm{His}^{+}$duplication strains isolated from crosses involving deletions his-2236 and his-2527. Deletion his-152 complements neither parental mutation and overlaps both (see Fig. 4 for its map position). All crosses were performed as described in Materials and Methods.

nt, not tested. 
recipient mutation complements neither of the duplication's parental deletions. The data in 'lable 2 indicate that none of 17 duplications tested is in either of these configurations.

Table 2 contains results of transductional crosses in which phenotypically His ${ }^{+}$ duplication strains are used as donors, and both of their parental his deletion mutants are used as recipients. For each duplication tested, transduction efficiency using either his-2236 or his-2527 as recipient is not significantly greater than that expected for a haploid donor carrying the appropriate parental deletion (see controls in the bottom three lines of Table 2). This suggests that none of the duplications is structured such that one of the deletions is cotransducible with the duplication junction. The low frequency of transductants observed is expected, since the donor strains harbor both deletion mutations, and both true recombinants and newly generated duplications can arise. These should occur at frequencies comparable to those obtained with haploid crosses between his-2236 and his-252\%.

When the same duplications tested above are used as donors in crosses with the large deletion his-152 (which overlaps both and complements neither parental mutation), no recombinants are formed. This demonstrates that none of the duplications is transducible in its entirety. The data in Table 2 are a first indication of the potentially large size of these transductional duplications.

One exception to the non-transducible nature of these duplications has been observed. When duplications are obtained from crosses involving deletions his-659 and his-152, the transductants usually segregate one parental mutation preferentially. In strains exhibiting this preferential segregation, the duplication state is often transducible. In Table 3 are shown segregation data for nine independent isolates of duplications involving these two mutations. In each case, preferential segregation of one parental mutation makes certain predictions about the duplication structure involved. For example, a duplication which preferentially retains his-152 should be

TABLE 3

Haploid parental segregants from duplications involving deletions his-659 and his. 152

\begin{tabular}{lrr}
\hline & \multicolumn{3}{c}{ Haploid segregant types } \\
Duplication & & $\%$ \\
strain & his.659 & his.152 \\
\hline TR3099 & $0(0 / 98)$ & $100(98 / 98)$ \\
TR3100 & $0(0 / 97)$ & $100(97 / 97)$ \\
TR3109 & $12(12 / 100)$ & $88(88 / 100)$ \\
TR3269 & $0(0 / 87)$ & $100(87 / 87)$ \\
TR3270 & $1(1 / 84)$ & $99(83 / 84)$ \\
TR3272 & $0(0 / 91)$ & $100(91 / 91)$ \\
TR3273 & $0(0 / 91)$ & $100(91 / 91)$ \\
TR3283 & $99(92 / 93)$ & $1(1 / 93)$ \\
TR3284 & $2(2 / 80)$ & $98(78 / 80)$ \\
\hline
\end{tabular}

Strains TR3099 through 3284 are independent duplication isolates from crosses between deletions his-659 and his-152. See Fig. 4 for the map position of these deletions. Extensively purified transductant clones were grown non-selectively and $\mathrm{H}_{\text {is }}$ - haploid parental segregants were identified as described in Materials and Methods. Figures in parentheses are the actual data. For each merodiploid, similar segregation results have been obtained in repeat experiments. 
structured such that deletion his-659 is much closer to the duplication junction (and therefore is more frequently lost) than deletion his.152. Most segregation events will excise the his-659 mutation and leave his-152 haploid segregants.

The transducibility of some of these duplications is demonstrated in Table 4. In most cases, duplication strains can act as efficient $\mathrm{His}^{+}$donors to only one of the parental deletion mutations; the resulting recombinants are unstable and have inherited the donor's duplication state. For each case of such transducible merodiploidy, the deletion that lies closest to the donor $s$ duplication junction (as judged by the preferential loss of that mutation during non-selective growth; see Table 3) has been transduced into a recipient carrying a complementing deletion. We interpret these as cases in which the duplication junction is being cotransduced with one (and only one) parental deletion. The transducibility of the merodiploid character of these exceptional strains supports the conclusion that these transductional duplications are in fact tandem chromosomal duplications.

TABle 4

Transducibility of a duplication state.

\begin{tabular}{|c|c|c|c|}
\hline \multirow{2}{*}{$\begin{array}{l}\text { Phage } \\
\text { donor }\end{array}$} & \multicolumn{3}{|c|}{$\begin{array}{c}\text { [Transductants/plaque-forming unit }] \times 10^{8} \\
\text { Recipient cells }\end{array}$} \\
\hline & his-1.52 & his-6.59 & his -3050 \\
\hline TR3099 & 41 & 0.5 & $0 \cdot 04$ \\
\hline TR3100 & 48 & 01.3 & $<0.02$ \\
\hline TR3109 & $0 \cdot 2$ & $01 \cdot 2$ & $<0.02$ \\
\hline TR3269 & 48 & 0.5 & $<0.02$ \\
\hline TR3270 & 57 & (1). 4 & $<0.02$ \\
\hline TR3272 & 48 & 0.4 & $<0.02$ \\
\hline TR3273 & 41 & 0.5 & $<0.02$ \\
\hline TR3283 & 0.6 & 57 & 0.04 \\
\hline TR3284 & 20 & 0.1 & $<0.02$ \\
\hline his-152 & $<0.02$ & 1.9 & $<0.02$ \\
\hline his-659 & $4 \cdot 2$ & $<0(1-0) 2$ & $<0.02$ \\
\hline LT-2 & 75 & 75 & 75 \\
\hline
\end{tabular}

Strains TR3099 thruugh 3284 are the sune as those whose segregation data are shown in Table 3 . Mutation his-3050 deletes the entire histidine operon. All crosses were performed as described in Materials and Methods.

\section{(j) Inclusion of nearby markers}

Certain insight into the mechanism of formation of these transductional duplications can be gained by mapping the lengths and end-points of the duplicated segments. To do this, we have utilized a genetic method used previously for mapping endpoints of $g l y T$ duplications (Hill \& Combriato, 1973). Figure 6 shows a diagram of the approach. Duplications can be generated in strains carrying nearby recessive auxotrophic markers-in this case met $G$. If the duplication does not include metG, the strain is haploid for metG- (this structure is shown in Fig. 6(a)). If the duplication includes metG, the strain is diploid for met $G^{-}$(this structure is shown in Fig. 6(b)). A functional met $G^{+}$allele may then be transduced into the strain. If the duplication does not include met $G$ (see Fig. 6(a)), this results in transduction of the single met $G^{-}$locus to $m e t G^{+}$. If the duplication includes metG (see Fig. 6(b)), this results in 
(a) Duplication does not include merG
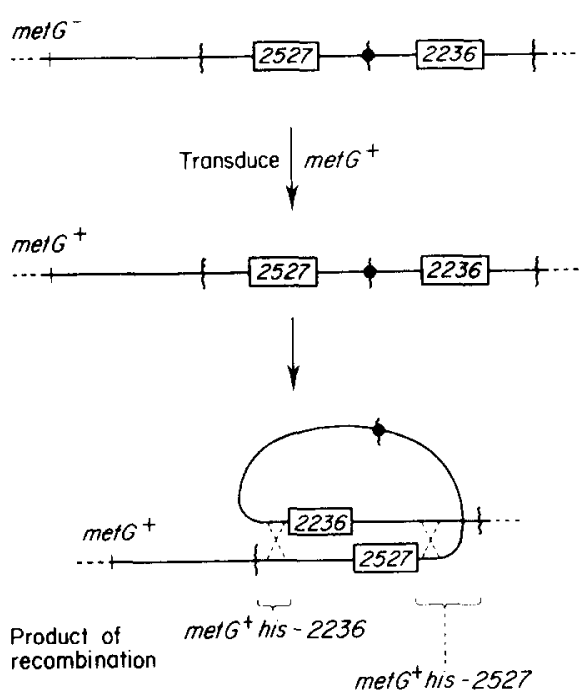

(b) Duplication includes meto
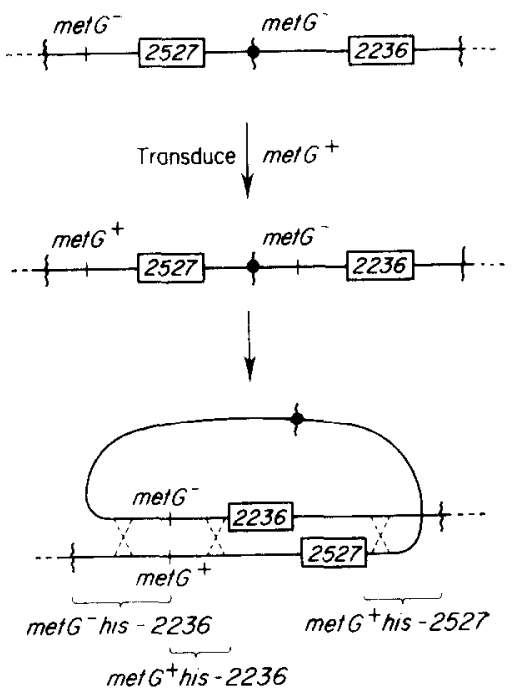

FiG. 6. A genetic method for detecting inclusion of genes within a tandemly duplicated region. (See text for details.)

transduction of either of the two met $G^{-}$loci (it doesn't matter which) to met $G^{+}$. The duplication may then be grown non-selectively and allowed to segregate. His ${ }^{-}$ haploid segregants may be picked and soored for their metf genotype. In a case where the duplication does not include metG (Fig. 6(a)), all His ${ }^{-}$segregants, whether they carry one parental his mutation or the other, must be met ${ }^{+}$(for the strain was haploid for $m e t G^{+}$). In a case where the duplication includes metG (Fig. 6(b)), however, haploid segregants which are met ${t^{-}}^{-}$will also appear. Met- segregants will arise from a $\mathrm{Met}^{+}$duplication strain. This indicates that the duplication strain was, in fact, heterozygous for met $G$, and that the duplication includes the met $G$ region.

Six independent duplications have been tested in this manner for inclusion of the nearby markers metG, pur $F$ and aroD (located 2, 10 and 10 minutes, respectively, clockwise from the histidine operon on the Salmonella map). Transductional duplications were generated in recipient strains multiply marked for these loci. Each recessive auxotrophic marker was individually transduced to prototrophy using a wild-type donor, and these potential heterozygotes were grown non-selectively. His - haploid segregants were picked and scored for the genotype of their his mutation and the appropriate marker. The data are presented in Table 5. Two of six duplications tested included all three nearby markers. Thus, the duplicated material in these strains extends at least ten minutes on the chromosomal map from the histidine operon. The remaining four duplications tested did not include even the closest marker tested, met $G$, two minutes removed from the histidine operon.

Thus it appears these transductional duplications can be quite large. 'The minimal size of the duplicated region for strains TR3215 and TR3287 is ten minutes (the distance between $h i s$ and the farthest marker tested, aroD). An estimate of the true duplication size may be obtained from the frequencies with which the four possible segregant genotypes arise following non-selective growth (see Fig. 6). If haploid 
TABLE 5

\section{Inclusion of nearby genes in the duplicated region}

\begin{tabular}{cccc}
\hline Duplication & $\begin{array}{c}\text { Percent auxotrophs among His } \\
\text { aro } D^{-}\end{array}$ & $\begin{array}{c}\text { haploid segregants } \\
\text { met } G^{-}\end{array}$ \\
\hline TR3215 & 79 & 72 & 20 \\
TR3287 & 11 & 9 & 21 \\
TR3216 & $<1$ & $<0.5$ & $<0.2$ \\
TR3217 & $<0.5$ & $<0.5$ & $<0.3$ \\
TR3288 & $<1$ & $<1$ & $<0.5$ \\
TR3289 & $<1$ & $<1$ & $<0.3$ \\
\hline
\end{tabular}

The above duplication strains were obtained in crosses using donor phage grown on deletion mutant his-2236 and recipient strain TR3168 (his-2527 aroD5 pur F145 metG319). Each duplica. tion strain was made $\mathrm{Met}^{+}, \mathrm{Aro}^{+}$or Pur ${ }^{+}$using phage grown on wild-type strainLT-2. After purification, these recombinants were grown non-selectively and scored for segregation of $\mathrm{His}^{-} \mathrm{Met}^{-}$, $\mathrm{His}^{-} \mathrm{Aro}^{-}$, or $\mathrm{His}^{-} \mathrm{Pur}^{-}$clones as described in the text.

segregants are generated by a random event between internally homologous regions, then the ratios of the frequencies of the various segregant types should reflect the ratios of the physical distances involved. By this method, estimates of the length of duplicated material in strain TR3215 range from 16 to 30 minutes (depending upon which nearby marker is scored), and from 15 to 28 minutes for strain TR3287.

\section{Discussion}

Among the progeny of many generalized transductional crosses within the histidine operon of Salmonella is a class of highly unstable merodiploid transductants. The data presented above suggest that these transductants can best be described as harboring tandem duplications of chromosomal material which includes the histidine operon, such that in each copy of the tandem duplication resides one of the two parental histidine mutations. That these transductants carry duplications of genetic material is supported by several of their properties. (1) They are unstable. Nonselective growth results in accumulation of $\mathrm{His}^{-}$parental segregants. (2) As in $E$. coli, their mucoidy is correlated with their diploidy. Strains which lose the diploid character (His ${ }^{-}$segregants) also lose the mucoid character simultaneously. (3) Complementation between parental mutations is required for their observation. (4) They may also be made heterozygous for genes adjacent to the histidine operon.

That both copies of the duplicated material are chromosomally located (as opposed to one copy being plasmid-borne) is supported by the fact that transductant instability is dependent upon a functional recombination system. Furthermore, agents which stimulate recombination activity (such as ultraviolet light) increase segregation frequencies (data not shown). Finally, that these transductants are in fact tandem chromosomal duplications is supported by the transducibility of the duplication state of some isolates.

The large size of duplicated material in these transductants has certain implications concerning the mechanism of their formation. Insertional mechanisms, whereby transducing particle DNA is somehow inserted into the recipient chromosome at or 
near its region of homology (possibly following circularization), are clearly unsatisfactory. The length of duplicated DNA in strains TR3215 and TR3287 is far greater than the amount which could be packaged by phage P22. We feel the data best support the mechanism outlined in Figure 7. This mechanism involves a low level of pre-existing tandem duplication in recipient chromosomes. such that among the population of recipient cells there are actually two classes: one class, the vast majority, being haploid for the histidine operon: and another small minority class, derived from and in equilibrium with the haploid, being tandemly diploid for the histidine operon. The transduction may proced in this partially diploid class simply by the formation of donor-type recombinants in one copy. If the donor in Figure 7 were one histidine deletion and the recipient were another complementing histidine deletion, formation

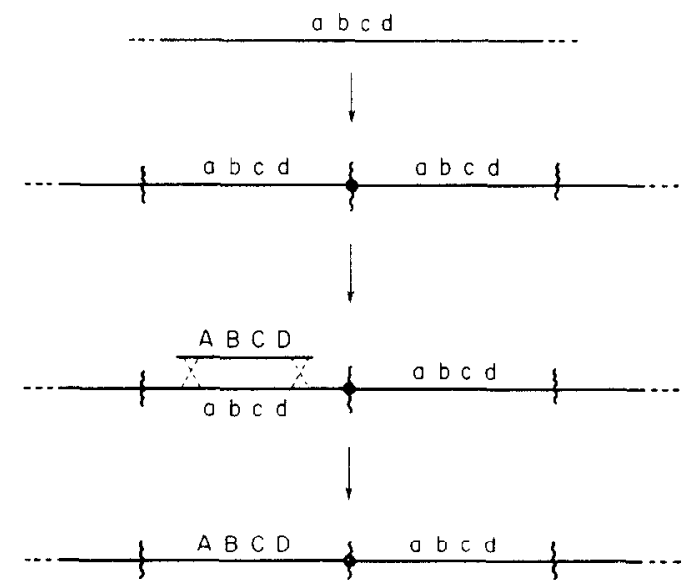

Fis. 7. A proposed mechanism for formation of transductional duplications involving reoombi. nation with one copy of a pre-existing tandem chromosomal duplication. Upper and lower case letters are non-genetic indications of hypothetical base sequences for donor and recipient DNAs respectively. Solid lines indicate double-stranded DNA. Dashed lines represent reciprocal recombinational events.

of donor types in either copy of the tandem duplication (and thus having no restrictions upon the placement of recombination events) would establish a complementing duplication such as those we observe. This mechanism appears most attractive in light of both the size of duplication involved and the location of parental mutations within the duplication. The data in Table 5 indicate that individual duplications may be as large as ten chromosomal map minutes, and the data in Table 2 indicate that most duplications are constructed such that neither parental mutation is cotransducible with the connecting point between duplicated regions. It is possible to envision alternative means of generating large tandem duplications during transduction. For each alternative, however, the duplication junction and one parental mutation must be cotransducible. The data in Table 2 indicate that this requirement is not met by 17 independent duplications.

For these reasons, we favor the model of transduction into a pre-existing tandem chromosomal duplication. If true, the frequency with which transductional duplications are observed should reflect the frequency of the duplication event itself. This estimate may be obtained by comparison of the yield of diploid transductants to the 
yield of total transductants when donor types are selected. Typically, duplication transductants arise approximately 0.1 to $0.01 \%$ as frequently as $\mathrm{His}^{+}$transductants using a wild-type phage donor. This is a rough approximation of the duplication frequency. A more direct estimate can be made from the observation that approximately $0.01 \%$ of total His ${ }^{+}$transductants using a wild-type phage donor are in fact duplications in which one copy is his ${ }^{+}$. Thus, duplications constitute a measurable fraction of total transductants, even without the use of tightly linked mutations. By these (admittedly rough) calculations, we estimate that tandem duplications of the histidine region are carried by $0 \cdot 1$ to $0 \cdot 01 \%$ of the cell population.

The contribution of phage infection to the duplication rates we observe is unknown. We use transduction as a method of trapping chromosomes in the duplicated state: therefore, phage infection and recombination are required for observation of the diploid character. If phage infection were to somehow increase the rate of duplication, our estimates would be artificially high. However, an independent estimate of the spontaneous duplication rate of the histidine operon has been obtained using a genedosage selective system which does not involve phage infection (Anderson \& Roth. manuscript in preparation). Amplification of the histidine operon may be observed by selection for increased his enzyme levels in strains which are unable to derepress the histidine operon. Under appropriate conditions. tandem his duplications are selected. Such duplications arise spontaneously at a frequency of approximately $10^{-4}$ per cell. This estimate of the spontaneous tandem duplication rate agrees well with the value obtained above.

Similar types of unstable transductants have been independently observed by others (Ely, 1973). Based upon transductional crosses within the histidine operon, they estimate that heterogenotes are formed with a frequency of approximately $0.03 \%$.

The mapping of duplication endpoints has not been extensive enough to permit us to conclude whether duplications have preferred endpoints. However, the observation that eight of nine duplications isolated from crosses involving his-152 and his-659 are structured such that one deletion is cotransducible with the duplication junction is suggestive that such non-randomness may exist for at least some strains. Duplications isolated from crosses involving these two deletions are unusual with regard to their preferential segregation of one parental genotype and the transducibility of the duplication state. Such preferential segregation has been observed in other duplication strains, but never so consistently as with the his-152/his169 pair. Furthermore, it does not matter which of these two mutations is used as the recipient during the cross. Such preferential segregation would be expected if either parental strain carried duplications with one endpoint near the histidine operon. Cotransduction of one his mutation with the duplication junction will re-establish the same duplication, provided a complementing his mutation is used as the recipient (see Fig. 5). Duplications present in the donor population will reappear if these conditions are met. Thus, the data suggest that either his- 752 or his- 659 (or both) is pre-disposed to the formation of duplications which have one endpoint near the histidine operon.

'The reasons for this pre-disposition are uncertain. Insertion sequence ISl (Starlinger \& Saedler, 1972) has been shown to greatly increase the frequency of deletion of nearby material (Reif \& Saedler, 1975). The same is true for IS3 in Salmonella (N. Kleckner, personal communication). In most cases the deletions so generated have one endpoint at the site of the IS insertion and a second endpoint at various 
sites. Deletion and duplication mutations are formally very similar events. Unequal recombination between sister chromatids would be expected to produce deletions and duplications as reciprocal products. Thus, it is attractive to speculate that strains his-152 or his-659 (or both) harbor an insertion sequence near the histidine operon. The study of tandem duplications may be one method of detecting IS-mediated unequal exchanges. Considering the large size of duplicated material, deletion mutations of comparable size should be lethal events.

Since the unstable transductants described here appear to arise in chromosomes which already harbor a tandem duplication, we have gained some insight into the duplication process itself. Specifically, duplications may be very large ( 7 to $21 \%$ of the Salmonella genome, depending upon which estimate is used) and may be very frequent. Tandem genetic duplications of nearly one third of the Salmonella chromosome have been reported elsewhere (Straus \& Hoffman, 1975). Thus, it appears Salmonella strains are able to tolerate quite large increases in their genome size, and may undergo such increases frequently.

We gratefully acknowledge the many helpful comments and criticisms of B. A. D. Stocker and J. Thorner during the preparation of this manuscript. This work was supported, in part, by a grant from the United States Public Health Service (GM-18663). One of us (R. P. A.) was supported by a National Science Foundation Pre-doctoral Fellowship.

\section{REFERENCES}

Botstein, D., Chan, R. \& Waddell, C. (1972). Virology, 49, 268-282.

Campbell, A. (1963). Virology, 20, 344-356.

Ely, B. (1973). Ph.D. thesis, Johns Hopkins University, Baltimore.

Fink, G., Klopotowski, 'T. \& Ames, B. (1967). J. Mol. Biol. 30, 81-95.

Folk, W. \& Berg, P. (1971). J. Mol. Biol. 58, 595-610.

Franklin, N. \& Luria, S. (1961). Virology, 15, 299-311.

Glansdorff, N. \& Sand, G. (1968). Genetics, 60, 257-268.

Hartman, P., Hartman, Z., Stahl, R. \& Ames, B. (1971). Advan. Genet. 16, 1-34.

Hill, C. \& Combriato, G. (1973). Mol. Gen. Genet. 127, 197--214.

Hill, C., Schiffer, D. \& Berg, P. (1969). J. Bacteriol. 99, 274 278.

Hilton, J., Kearney, P. \& Ames, B. (1965). Arch. Biochem. Biophys. 112, 544-547.

Jacob, F. \& Monod, J. (1961). Cold Spring Harbor Symp. Quant. Biol. 26, 193-211.

Levine, M. (1972). Current Topics in Microbiol. \& Immunol. 58, 135-156.

Lew, K. \& Roth, J. (1970). Virology, 40, 1059-1062.

Miller, C. \& Roth, J. (1971). J. Mol. Biol. 59, 63-75.

Parma, D., Ingraham, L. \& Snyder, M. (1972). Genetics, 71, 319 335.

Reif, H. J. \& Sredler, H. (1975). Mol. Gen. Genet. 137, 17-28.

Sanderson, K. (1972). Bacteriol. Rev. 36, 558-586.

Silverman, M. \& Simon, M. (1973). J. Bacteriol. 113, 105113.

Smith, H. \& Levine, M. (1967). Virology, 31, 207-216.

Starlinger, P. \& Saedler, H. (1972). Biochimie, 54, 177.

Stodolsky, M. (1973). Virology, 53, 471-475.

Straus, D. \& Hoffman, G. (1975). Genetics, 80, 227-237.

Sturtevant, A. (1925). Genetics, 10, 117-147.

Vogel, H. \& Bonner, D. (1956). J. Biol. Chem. 218, 97--106.

Zinder, N. (1958). Virology, 5, 291-326. 